\title{
Improved Outcomes Associated With the Use of Intravenous Acetaminophen for Management of Acute Post-Surgical Pain in Cesarean Sections and Hysterectomies
}

\author{
Richard D. Urman ${ }^{\mathrm{a}}$, Elaine A. Boing ${ }^{\mathrm{b}}$, An T. Pham ${ }^{\mathrm{c}}$, Victor Khangulov ${ }^{\mathrm{d}}$, Randi Fain ${ }^{\mathrm{e}}$, \\ Brian H. Nathanson ${ }^{\text {f }}$ Xuan Zhang ${ }^{\mathrm{d}}$, George J. Wan ${ }^{\mathrm{b}}$, \\ Belinda Lovelace ${ }^{\mathrm{b}}$, Jessica Cirillo ${ }^{\mathrm{b}, \mathrm{g}}$
}

\begin{abstract}
Background: Post-surgical pain impacts many patient outcomes. Effective pain management increasingly relies on multimodal analgesia regimens in which acetaminophen (APAP) is a key component. The aim of our study was to examine the impact of oral APAP versus intravenous (IV) APAP as a component of post-surgical pain management after Cesarean sections and hysterectomies.

Methods: A retrospective analysis of the Cerner HealthFacts $\AA$ database (from January, 2011 to December, 2015) was conducted to compare outcomes of Cesarean section and hysterectomy surgery patients who received oral APAP to those who received IV APAP post-surgically. Length of stay (LOS), daily morphine milligram equivalent (MME) consumption, the presence of potential opioid-related adverse events (ORADEs), and total pharmacy costs were assessed. Adjusted results were derived using inverse probability weighted regression adjustment (IPW-RA) estimators based on covariates that included demographics, comorbidities, patient clinical characteristics, and hospital characteristics.
\end{abstract}

Results: The study identified 29,124 Cesarean section patients (24,612 oral APAP; 4,512 IV APAP) and 9,767 hysterectomy surgery patients (5,586 oral APAP; 4,181 IV APAP). Compared to the oral APAP group, the IV APAP group had reductions in adjusted LOS (Cesarean section: $-11.7 \%$ days $(\mathrm{P}<0.001)$, hysterectomy: $-11.8 \%$ days

Manuscript submitted February 8, 2018, accepted February 26, 2018

${ }^{a}$ Harvard Medical School and Brigham and Women's Hospital, Boston, MA, USA

${ }^{b}$ Health Economics and Outcomes Research Department, Mallinckrodt Pharmaceuticals, Bedminster, NJ, USA

'School of Pharmacy, University of California San Francisco, San Francisco, CA, USA. This author was an employee of Mallinckrodt Pharmaceuticals during the conduct of this study

dBoston Strategic Partners, Inc., Boston, MA, USA

'Medical Affairs Department, Mallinckrodt Pharmaceuticals, Bedminster, NJ, USA

fOptiStatim, LLC, Longmeadow, MA, USA

gCorresponding Author: Jessica M. Cirillo, 1425 U.S. Route 206, Bedminster, NJ 07921, USA. Email: jessica.cirillo@mallinckrodt.com

doi: https://doi.org/10.14740/jocmr3380w
$(\mathrm{P}=0.005))$, lowered adjusted daily MME consumption from day 0 to day 3 (Cesarean section: $-1.6 \mathrm{mg}(\mathrm{P}<0.001)$, hysterectomy: -1.7 $\mathrm{mg}(\mathrm{P}=0.014)$ ), and reduced risk of ORADEs for Cesarean sections (relative risk of $0.45, \mathrm{P}<0.001$ ). Total pharmacy costs were not significantly different between the two APAP groups.

Conclusions: Post-surgical pain managed with IV APAP in patients undergoing Cesarean section or hysterectomy was associated with shorter LOS, reduced risk of ORADEs, and lower opioid consumption compared to patients managed with oral APAP, without adversely impacting total pharmacy costs.

Keywords: IV acetaminophen; Multimodal analgesia; Cesarean section; Hysterectomy; Opioid; Post-surgical pain; Acute pain; Health outcomes

\section{Introduction}

Post-surgical pain management is the key to successful surgical recovery [1]. Acute pain impacts short-term and long-term post-surgical outcomes since inadequate pain management can result in extended hospital length of stay (LOS) and increased risk of developing chronic pain $[2,3]$. Population-based studies suggest that women may be more likely to experience chronic pain syndromes, and obstetric and gynecologic surgeries are among the most common surgeries in women [4-6].

The most commonly used analgesics for post-surgical pain are intravenous (IV) and oral opioids. While effective for pain relief, opioids are associated with a variety of costly opioid-related adverse events (ORADEs) [7, 8]. Opioid use can lead to addiction and abuse [9], and the overprescribing of opioids post-surgery is a contributor to the opioid epidemic in the United States [10]. Differences in opioid efficacy have also been reported between women and men $[11,12]$, and following Cesarean deliveries, additional concerns for postoperative pain management arise. For example, although breastfeeding while receiving opioid medications is generally permitted [13, 14], sedation associated with opioid-based analgesia following Cesarean sections can disrupt breastfeeding, infant care, and mother-infant bonding [15]; while suboptimal pain manage- 
ment or increased sedation may impair the mother's ambulation, which increases the risk of thromboembolic disease [16].

New guidelines from the American Pain Society (APS), the American Society of Anesthesiologists (ASA), and the American Society of Regional Anesthesia and Pain Medicine (ASRA), as well as other professional societies, strongly recommend the use of multimodal therapies, incorporating non-opioid analgesics as foundational therapies to treat postoperative pain [17]. The American College of Obstetricians and Gynecologists (ACOG), the Society for Maternal-Fetal Medicine (SMFM), and the Academy of Breastfeeding Medicine (ABM) have specific guidelines and recommendations for breastfeeding mothers that include maximizing the use of multi-modal non-opioid analgesics, such as non-steroidal anti-inflammatory drugs (NSAIDs) and acetaminophen (APAP) if feasible and as appropriate [18]. Multimodal analgesia (MMA) regimens can generally reduce the use of opioids and consequent ORADEs [17]. Since most pain is multi-factorial in nature, owing to the physiology of pain transmission, the use of combinations of analgesics with differing mechanisms of action may also have additive or synergistic effects to reduce pain [19-22].

MMA regimens are well-established in guidelines for both Cesarean section and hysterectomy surgeries. In both surgical types, MMA has been associated with reduced hospital LOS and decreased opioid use [23-26]. A plethora of medications are utilized in MMA regimens, including NSAIDs, nonNSAIDs (e.g. APAP) and GABA analogs (gabapentin, pregabalin) $[20,21]$. However, NSAIDS may be contra-indicated when certain comorbidities are present [27].

Oral APAP has been widely used for over 50 years. An intravenous (IV) APAP formulation appropriate for post-surgical patients who cannot take oral drugs due to sedation, nausea, vomiting, or slow gastrointestinal (GI) recovery became available in 2010. Use of IV APAP has expanded in several surgery types, including orthopedic and spine surgeries, but the overall impact of IV APAP on post-surgical pain remains relatively unstudied in non-clinical trials, real-world conditions, and especially in obstetric or gynecologic surgeries $[2,28,29]$. The primary objective for this retrospective analysis of electronic health records (EHR) was to evaluate the clinical and administrative outcomes associated with the utilization of oral APAP versus IV APAP in women undergoing a Cesarean section or hysterectomy.

\section{Methods}

\section{Study design}

This retrospective cohort study was conducted using data from the Cerner HealthFacts ${ }^{\circledR}$ dataset (Cerner HealthFacts ${ }^{\circledR}$; Kansas City, MO) from January 1, 2011 to December 31, 2015. Cerner HealthFacts ${ }^{\circledR}$ is a large US electronic health database, based on patient records across the United States from over 600 hospitals. Patients receiving oral APAP versus IV APAP (verified through the selected National Drug Codes; NDC) and medication administration route in a post-surgical setting were evaluated. The study was granted an Institutional Review Board exemption from Western IRB.

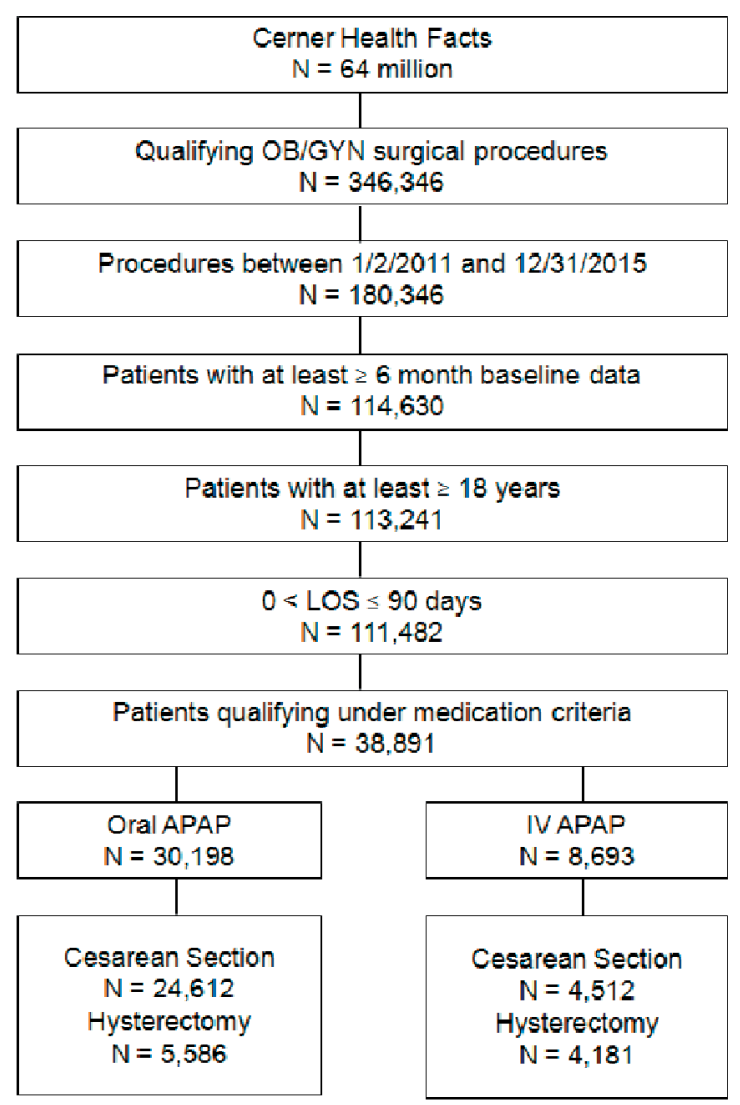

Figure 1. Patient selection in OB/GYN patients treated with oral vs. IV APAP. The study identified 30,198 unique Cesarean section and hysterectomy patients who were treated with oral APAP and 8,693 Cesarean section and hysterectomy patients who were treated with IV APAP. IV: intravenous; APAP: acetaminophen; OB/GYN: obstetrics and gynecology.

\section{Patients and selection}

The patient population was selected using International Classification of Diseases (ICD)-9-CM and ICD-10-CM codes for Cesarean section and hysterectomy surgeries (Fig. 1). Inclusion criteria selected adult patients ( $\geq 18$ years) undergoing a qualifying Cesarean or hysterectomy procedure (see Supplementary Table 1, www.jocmr.org), with admission between January 1, 2011 and December 31, 2015, and administration of either oral APAP or IV APAP during the first $24 \mathrm{~h}$ post-surgery (day 0) (see medication criteria schematic in Supplementary Figure 1, www.jocmr.org). Oral APAP was defined as tablet or capsule formulations of APAP. Other formulations, such as liquid taken orally or suppositories, were not considered for inclusion. Combination APAP medications (e.g. acetaminophen/ dextromethorphan/doxylamine succinate/pseudoephedrine $\mathrm{HCl}$ ) were not regarded as oral APAP for inclusion purposes. Exclusion criteria included patients with $<6$ months of history captured in the database prior to the index visit, missing information for age or gender, and in-hospital LOS $>90$ days. The oral APAP and IV APAP cohorts were mutually exclusive. Patients in the oral APAP cohort could not have received IV 
Table 1. Demographics and Baseline Characteristics for Cesarean Section Surgery Patients

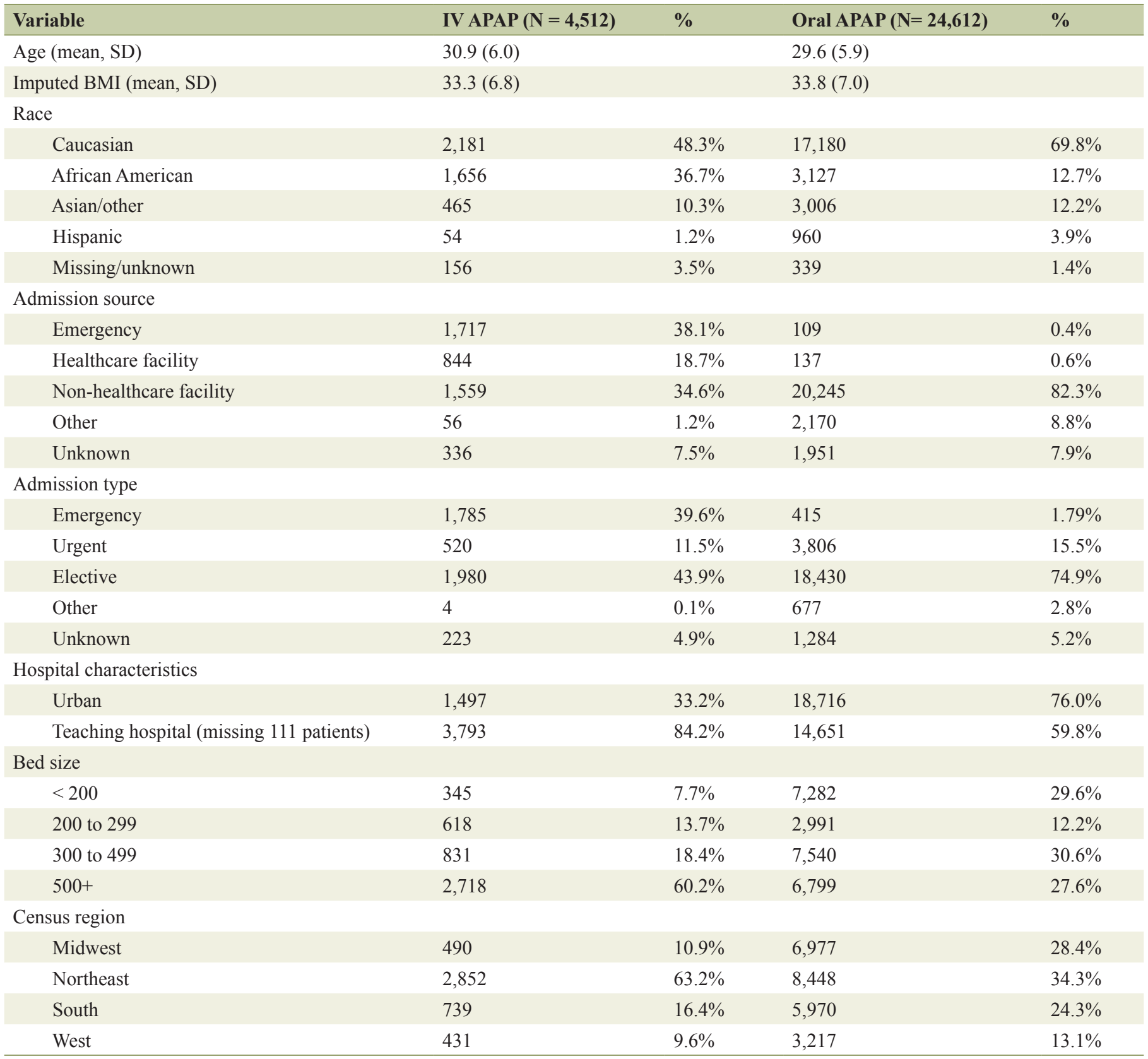

A total of 29,124 Cesarean section surgery patients were analyzed. IV: intravenous; APAP: acetaminophen; SD: standard deviation; BMI: body mass index.

APAP on days 0 - 3 and vice versa. Patients were excluded if they had received either oral or IV APAP on day 4 or after (see medication criteria schematic in Supplementary Figure 1, www.jocmr.org).

\section{Patient characteristics}

Baseline characteristics (e.g. age, gender, race, year of admission) and chronic comorbidities (based on the definitions of
Elixhauser) at index admission and study outcomes were collected [30]. The Elixhauser comorbidities were calculated using the approach from Healthcare Cost and Utilization Project (HCUP), Elixhauser Comorbidity Software, Version 3.7 [30]. Hospital characteristics (e.g. bed size, urban/rural status, census region) at index admission and payer type were collected as reported. Additional information about procedure type for hysterectomies were incorporated into analytical models as open, laparoscopic, total vaginal hysterectomy, and total abdominal hysterectomy. 


\section{Study outcomes}

Study outcomes included length of hospital stay, presence of ORADEs, total daily opioid consumption (i.e. morphine (mg) equivalent; MME) and total pharmacy cost. Opioid consumption was calculated using the amounts administered to the patient, while taking into account any doses that were returned to the hospital pharmacy unused. The conversion to MME was performed as previously described [31, 32]. For outpatients, total, per patient, pharmacy cost was calculated by summing unit costs for all medications that were dispensed (unit cost represent cost per medication package), and for inpatients, it was calculated by summing unit cost of the medication multiplied by the number of medication units that were charged to the pharmacy (unit cost represents cost per unit). Missing body mass index (BMI) values were imputed with a univariate imputation sampling method from a regression model of known covariates [33]. To minimize the effect of outliers, total pharmacy costs were Winsorized at the $2.5 \%$ level, which means that values above the 97.5 th percentile were set to the 97.5 th percentile value and values below the 2.5 th percentile were set to the 2.5 th percentile value. Total morphine equivalent dosing had the following constraints to be deemed valid: No 4-day total from Day 0 to Day 3 could exceed $800 \mathrm{mg}$ MME and each day after Day 0 could have no more than $200 \mathrm{mg}$ MME.

\section{Statistical analysis}

The Cesarean and hysterectomy patients were analyzed separately using multivariable methods. The primary method to estimate the impact of IV APAP on the study outcomes (e.g. hospital LOS) was inverse probability weighted regression adjustment (IPW-RA) with covariates that included patient demographics, comorbidities, patient admission type (e.g. emergency admission), relevant surgical characteristics (e.g. a laparoscopic hysterectomy), and prior drug history up to the day of surgery [34, 35]. See Supplementary Tables 2, 3 (www.jocmr.org) for all variables used for multivariable adjustment. Hospital characteristics (e.g. the number of beds, teaching status, geographic location) were also used for adjustment.

As a sensitivity analysis, generalized linear models (GLM) estimated the effect of IV APAP on the outcomes and were used with the same covariates that were used to estimate the propensities in the IPW-RA model. For continuous outcomes (e.g. LOS) a logarithmic link and a gamma distribution were used in the regression models. When the outcomes were binary, logistic regression models were utilized. Odds ratios (ORs) were converted to risk ratios (relative risks (RRs)) [36]. All GLM models used robust, Huber-White standard error estimators, clustered at the hospital level to account for potential serial correlation of patient outcomes among patients treated in the same hospital.

\section{Results}

We identified 38,891 patients, with 9,767 (25.1\%) undergoing hysterectomies and 29,124 (74.9\%) patients undergoing Cesar- ean section surgery (Tables 1, 2). Overall, 8,693 (22.4\%) of all Cesarean section and hysterectomy surgical patients received IV APAP (Tables 1, 2). However, the proportion varied by surgical type, with $15.5 \%$ of the Cesarean patients and $42.8 \%$ of hysterectomy patients receiving IV APAP $(\mathrm{P}<0.001)$.

Mean ages within surgical categories were similar between the oral and IV APAP groups (Cesarean mean age $=29.6$ vs. 30.9 years; hysterectomy mean age $=47.1$ vs. 47.4 years) $($ Tables 1,2$)$. The majority of hysterectomy patients $(90.5 \%$ of oral APAP and $64.3 \%$ of IV APAP) were treated at urban facilities. In contrast, $76.0 \%$ of Cesarean section patients in the oral APAP cohorts were treated at urban facilities and only $33.2 \%$ of the IV APAP cohorts were treated at urban facilities. Regardless of procedure type (Cesarean section or hysterectomy) or APAP cohort (oral or IV), the majority of patients were treated at teaching hospitals, with a greater percentage of IV APAP cohorts treated at a teaching hospital versus the oral APAP cohorts.

Adjusted results (see (Supplementary Table 4, www.jocmr. org) for unadjusted outcomes) were derived using IPW-RA estimators based on covariates that included demographics, comorbidities, patient clinical characteristics, and hospital characteristics. In the IPW-RA analyses (Table 3), Cesarean section patients receiving IV APAP (versus oral APAP) showed an $11.7 \%$ shorter LOS. In the IV APAP cohort, the total (marginal) mean adjusted LOS was 3.40 days $(95 \%$ CI 3.29, 3.51) for IV APAP versus 3.85 days $(95 \%$ CI 3.60, 4.10$)$ for oral APAP. This 0.45 day shorter LOS for IV APAP patients (95\% CI -0.72, -0.17) was statistically significant $(\mathrm{P}<0.001)$. Similarly, in patients undergoing hysterectomies, IV APAP was associated with an $11.8 \%$ shorter LOS, with a total adjusted 2.01 days $(95 \%$ CI 1.89, 2.13) for IV APAP versus LOS of 2.28 days $(95 \%$ CI 1.14, 2.43) for oral APAP. This 0.27 day shorter LOS for IV APAP patients (95\% CI -0.46, -0.08) was also statistically significant $(\mathrm{P}<0.001)$. The results in the GLM sensitivity analyses (Table 3 ) showed similar reductions in LOS associated with the receipt of IV APAP.

Total MME consumption per day observed from Day 0 to Day 3 was statistically significantly lower for IV APAP patients in both Cesarean section and hysterectomy surgeries $(-1.6 \mathrm{mg} ; 95 \% \mathrm{CI}-2.5,-0.8 ; \mathrm{P}<0.001$ for Cesarean section and $-1.7 \mathrm{mg} ; 95 \%$ CI $-3.1,-0.3 ; \mathrm{P}<0.014$ for hysterectomy) (Table 3). However, these differences in total MME consumption per day were not significantly different by APAP route in the GLM sensitivity analysis for either surgery type.

IV APAP was associated with fewer potential ORADEs [37] than oral APAP in Cesarean section surgeries (relative risk $=0.45 ; 95 \%$ CI $0.26,0.78 ; \mathrm{P}<0.001)$. However, there was no significant association between the route of APAP administration and the adjusted risk of potential ORADEs in hysterectomy surgeries (relative risk $=1.09 ; 95 \%$ CI $0.77,1.41 ; \mathrm{P}=$ 0.57 ). The findings in the GLM sensitivity analyses were also concordant, with significant risk reduction for Cesarean section, and no significant association for hysterectomy.

IV APAP and oral APAP showed no significant difference in pharmacy costs for hysterectomy patients. The IPW-RA model did not converge for Cesarean section patients, possibly due to some predictors being uncommon but correlated with both treatment and costs. Moreover, total pharmacy costs were not significantly different for IV APAP and oral APAP in either surgery in the GLM sensitivity analysis. We also examined 
Table 2. Demographics and Baseline Characteristics for Hysterectomy Surgery Patients

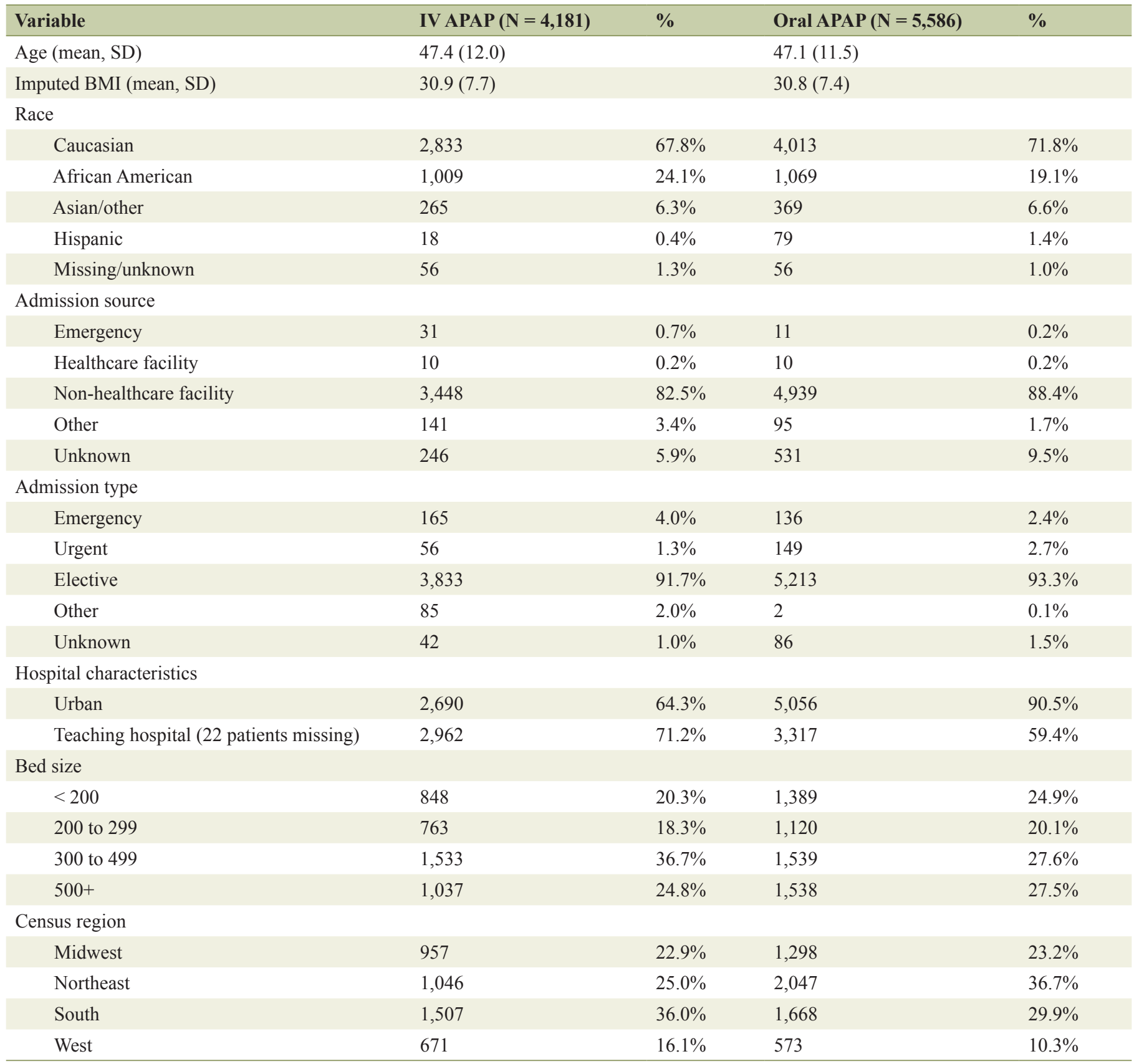

A total of 9,767 hysterectomy surgery patients were analyzed. IV: intravenous; APAP: acetaminophen; SD: standard deviation; BMI: body mass index.

other known cost drivers, including 90-day readmissions, ICU admissions and mortality. ICU admissions showed no significant differences between IV APAP and oral APAP use in both surgery types and the other outcomes were too rare to model reliably in these populations (data not reported).

\section{Discussion}

Using a large, multi-center, retrospective database, we dem- onstrated that IV APAP use is associated with significantly shorter LOS (by approximately 12\%) in Cesarean section and hysterectomy patients when compared to oral APAP. This analysis is one of the first to assess the clinical outcomes and costs of oral APAP versus IV APAP from EHRs, from a robust database of over 600 hospitals and over 62 million patients. Our study focused on Cesarean sections and hysterectomies, where reductions in LOS may be most impactful for hospital costs and resources, given that these patients generally have consistent LOS, with less variation when compared to other surgeries 


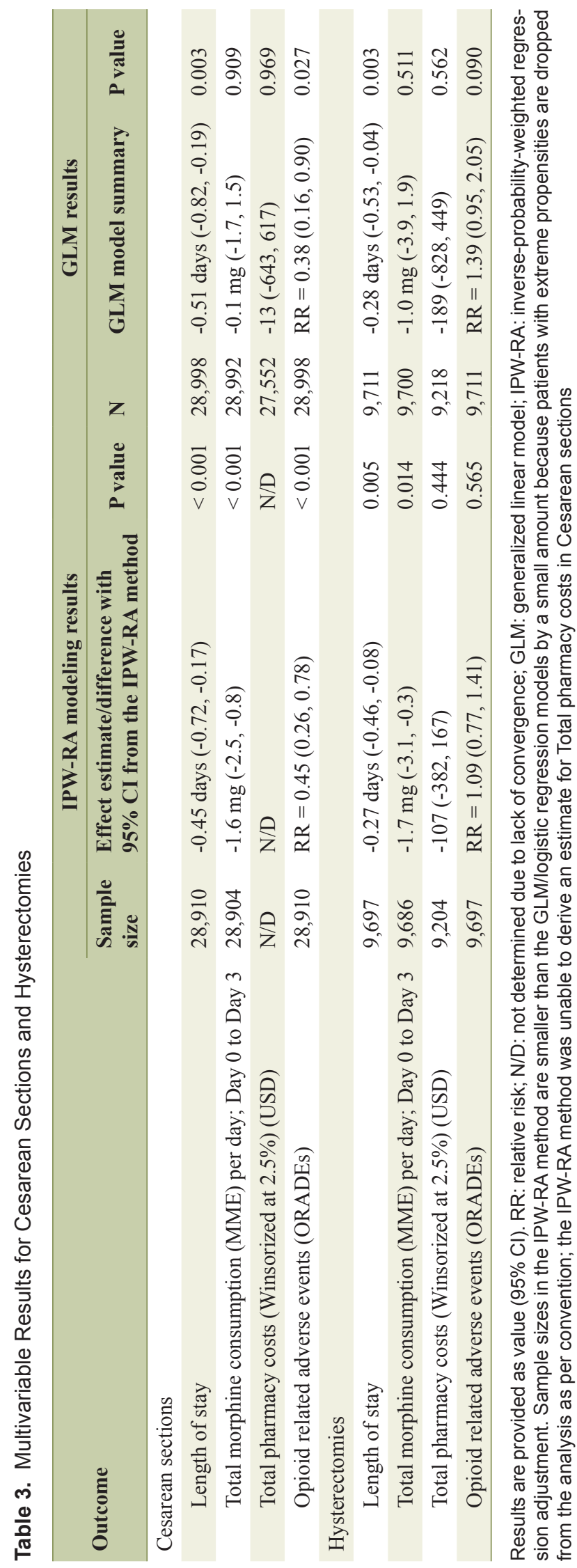

requiring hospitalization. Additionally, research indicates that pain management may differ by gender [5], but the potential for confounding by sex is eliminated by focusing on Cesarean sections and hysterectomies.

A previous claims-based study of Cesarean section and hysterectomy surgeries showed significantly less daily opioid use and lower hospitalization costs in patients receiving IV APAP as part of MMA versus patients receiving IV opioid-only analgesia in Cesarean section and a non-significant trend towards less opioid use and lower cost in hysterectomy; however, the claims-based analysis did not show reductions in LOS in the IV APAP cohort following adjustment [24]. The current study demonstrates statistically significant reductions in LOS and opioid consumption for both Cesarean section and hysterectomy patients. The LOS reductions approached $12 \%$, translating to a reduction of approximately 8 to $12 \mathrm{~h}$, or potentially one nursing shift per day. In the Cesarean group, IV APAP patients also had lower risk of developing an ORADE. There were no statistically significant differences in total pharmacy costs between oral APAP and IV APAP, despite cost differences between these formulations.

These findings are consistent with prior studies of MMA protocols including IV APAP, demonstrating a reduced LOS in orthopedic and spine surgery patients $[38,39]$. Retrospective studies in orthopedic and other surgery types have found similar associations [24, 40-42]. MMA is thought to improve surgical outcomes by reducing opioid dosing, resulting in decreased ORADEs and complications, and creating synergies that result in pain reductions. APAP exerts its effects in the central nervous system, and versus oral APAP, IV APAP has a faster T max and a higher $\mathrm{C}$ max, with pharmacokinetics that achieve greater concentration in cerebrospinal fluid, suggesting better analgesia from this formulation [43, 44]. Our results support these findings and suggest that IV APAP use may be cost-effective for Cesarean section and hysterectomy surgeries through the reduction of LOS, opioid consumption, and ORADEs.

Other studies have also found that MMA following Cesarean sections or hysterectomies can help to minimize/manage postoperative pain and drive positive clinical outcomes [26, 45]. MMA after Cesarean section has been shown to provide better clinical outcomes with less reliance on opioid pain medications $[45,46]$. Supplemental uses of non-opioid medications have been associated with superior pain relief, improved analgesia, and opioid sparing (reduced morphine consumption) [47, 48].

Similar to Cesarean section surgeries, MMA in hysterectomy surgeries is associated with reduced hospital LOS following both open abdominal surgeries and minimally invasive/ laparoscopic surgeries [23, 26]. Likewise, MMA in hysterectomy surgeries decreased total opioid utilization perioperatively on postoperative days 1 and 2 [25]. Furthermore, this reduced opioid consumption via MMA does not reduce the efficacy of pain relief [25].

While our results demonstrate an association between IV APAP and shorter LOS, further studies should examine other aspects of efficacy, including pain reduction. Additionally, multimodal therapy including IV APAP may help to reduce sedation associated with opioids to promote mother-baby bonding, caretaking, breast-feeding, and ambulation $[15,16]$. Furthermore, reducing the LOS and ORADEs could also help 
mothers return to their families earlier and perform their daily activities normally. Therefore, using IV APAP in Cesarean section surgeries may benefit both the mother and the newborn infant.

Using IV APAP in a multimodal pain management approach after Cesarean sections or hysterectomies may also reduce the risk of postoperative opioid-related complications by decreasing the amount of opioids used. In fact, a previous study found that, compared with IV opioid-only analgesia, managing pain after Cesarean sections or hysterectomies with the addition of IV APAP decreased both opioid use and hospitalization costs [24].

The study's strengths include the large, multi-center nature of the database and the breadth of variables used to adjust for potential confounding. Another strength is the statistical methodology; IPW-RA is a more sophisticated method than related approaches like propensity score matching. With IPWRA, one model is used to derive probabilities (propensities) for treatment and another uses these probabilities in a weighted regression model to estimate average treatment effects. Because IPW-RA estimators are doubly robust, only one of the two models must be correctly specified for the IPW-RA estimator to be consistent. IPW-RA models may not produce a solution with a large number of rare predictors. To address this possibility, we used a more traditional regression model approach as a sensitivity analysis and found generally similar findings.

One limitation of this study is that these findings may not be generalizable to Cesarean or hysterectomy patients that differ substantially from those observed in the Cerner HealthFacts database. It is also unknown how oral APAP versus IV APAP correlates with hospital LOS in other surgical populations. Another study limitation is unmeasured confounding. Laboratory results and acuity measures were not analyzed, and the chronic comorbidities defined by Elixhauser were not developed specifically for the Cesarean section and hysterectomy populations [30]. Specific Cesarean section and hysterectomy variables like pre-eclampsia were not available for this analysis. Nevertheless, a large number of covariates were used for multivariable adjustment. Finally, total cost data could not be reported, as the percentage of patients missing unadjusted data was too high.

Overall, this study provides further evidence supporting the preferential use of IV APAP as part of MMA regimens in Cesarean section and hysterectomy surgeries by demonstrating decreases in LOS, opioid consumption, and ORADE rate.

\section{Acknowledgments}

This study was supported by Mallinckrodt Pharmaceuticals. The authors thank Jeffrey R. Skaar and Anna K. Talaga at Boston Strategic Partners, Inc. for editorial support.

\section{Conflict of Interest}

EAB, RF, GJW, and JC are employees of Mallinckrodt Pharmaceuticals, and BL and ATP were employees of Mallinck- rodt Pharmaceuticals at the time of the study. VK and $\mathrm{XZ}$ are consultants with Boston Strategic Partners, Inc., which contracted with Mallinckrodt Pharmaceuticals for this study. BHN is an employee of OptiStatim, LLC and was contracted for this study by Boston Strategic Partners, Inc.

\section{References}

1. Gan TJ, Robinson SB, Oderda GM, Scranton R, Pepin J, Ramamoorthy S. Impact of postsurgical opioid use and ileus on economic outcomes in gastrointestinal surgeries. Curr Med Res Opin. 2015;31(4):677-686.

2. Hansen RN, Pham AT, Boing EA, Lovelace B, Wan GJ, Miller TE. Comparative analysis of length of stay, hospitalization costs, opioid use, and discharge status among spine surgery patients with postoperative pain management including intravenous versus oral acetaminophen. Curr Med Res Opin. 2017;33(5):943-948.

3. Reddi D, Curran N. Chronic pain after surgery: pathophysiology, risk factors and prevention. Postgrad Med J. 2014;90(1062):222-227; quiz 226.

4. Yu HY, Tang FI, Yeh MC, Kuo BI, Yu S. Use, perceived effectiveness, and gender differences of pain relief strategies among the community-dwelling elderly in Taiwan. Pain Manag Nurs. 2011;12(1):41-49.

5. Pieretti S, Di Giannuario A, Di Giovannandrea R, Marzoli F, Piccaro G, Minosi P, Aloisi AM. Gender differences in pain and its relief. Ann Ist Super Sanita. 2016;52(2):184189.

6. Discharges with at least one procedure in nonfederal short-stay hospitals, by sex, age, and selected procedures: United States, selected years 1990 through 2009-2010. National Center for Health Statistics. Hyattsville, MD. 2015.

7. Kane-Gill SL, Rubin EC, Smithburger PL, Buckley MS, Dasta JF. The cost of opioid-related adverse drug events. J Pain Palliat Care Pharmacother. 2014;28(3):282-293.

8. Oderda GM, Said Q, Evans RS, Stoddard GJ, Lloyd J, Jackson K, Rublee D, et al. Opioid-related adverse drug events in surgical hospitalizations: impact on costs and length of stay. Ann Pharmacother. 2007;41(3):400-406.

9. Baldini A, Von Korff M, Lin EH. A review of potential adverse effects of long-term opioid therapy: a practitioner's guide. Prim Care Companion CNS Disord. 2012;14(3).

10. Kelly MA. Current postoperative pain management protocols contribute to the opioid epidemic in the United States. Am J Orthop (Belle Mead NJ). 2015;44(10 Suppl):S5-8.

11. Fillingim RB, King CD, Ribeiro-Dasilva MC, RahimWilliams B, Riley JL, 3rd. Sex, gender, and pain: a review of recent clinical and experimental findings. J Pain. 2009;10(5):447-485.

12. Fillingim RB, Gear RW. Sex differences in opioid analgesia: clinical and experimental findings. Eur J Pain. 2004;8(5):413-425.

13. Wanderer JP, Rathmell JP. Anesthesia \& breastfeeding: more often than not, they are compatible. Anesthesiology 2017;127:A15. 
14. Cobb B, Liu R, Valentine E, Onuoha O. Breastfeeding after anesthesia: a review for anesthesia providers regarding the transfer of medications into breast milk. Transl Perioper Pain Med. 2015;1(2):1-7.

15. Howie WO, McMullen PC. Breastfeeding problems following anesthetic administration. J Perinat Educ. 2006;15(3):50-57.

16. Gadsden J, Hart S, Santos AC. Post-cesarean delivery analgesia. Anesth Analg. 2005;101(5 Suppl):S62-69.

17. Chou R, Gordon DB, de Leon-Casasola OA, Rosenberg JM, Bickler S, Brennan T, Carter T, et al. Management of postoperative pain: a clinical practice guideline from the American Pain Society, the American Society of Regional Anesthesia and Pain Medicine, and the American Society of Anesthesiologists' Committee on Regional Anesthesia, Executive Committee, and Administrative Council. J Pain. 2016;17(2):131-157.

18. The American College of Obstetricians and Gynecologists (ACOG) tSfM-FMS, and the Academy of Breastfeeding Medicine (ABM). practice advisory on codeine and tramadol for breastfeeding women. 2017.

19. Raffa R. Pharmacological aspects of successful long-term analgesia. Clin Rheumatol. 2006;25(Suppl 1):S9-15.

20. Raffa RB, Clark-Vetri R, Tallarida RJ, Wertheimer AI. Combination strategies for pain management. Expert Opin Pharmacother. 2003;4(10):1697-1708.

21. Raffa RB. Pharmacology of oral combination analgesics: rational therapy for pain. J Clin Pharm Ther. 2001;26(4):257-264.

22. Woolf CJ, American College of P, American Physiological S. Pain: moving from symptom control toward mechanism-specific pharmacologic management. Ann Intern Med. 2004;140(6):441-451

23. Santoso JT, Ulm MA, Jennings PW, Wan JY. Multimodal pain control is associated with reduced hospital stay following open abdominal hysterectomy. Eur J Obstet Gynecol Reprod Biol. 2014;183:48-51.

24. Hansen RN, Pham AT, Lovelace B, Balaban S, Wan GJ. Comparative analysis of inpatient costs for obstetrics and gynecology surgery patients treated with IV acetaminophen and IV opioids versus IV opioid-only analgesia for postoperative pain. Ann Pharmacother. 2017;51(10):834839.

25. Herring BO, Ader S, Maldonado A, Hawkins C, Kearson M, Camejo M. Impact of intravenous acetaminophen on reducing opioid use after hysterectomy. Pharmacotherapy. 2014;34(Suppl 1):27S-33S.

26. Wong M, Morris S, Wang K, Simpson K. Managing postoperative pain after minimally invasive gynecologic surgery in the era of the opioid epidemic. J Minim Invasive Gynecol. 2017.

27. Moore N, Pollack C, Butkerait P. Adverse drug reactions and drug-drug interactions with over-the-counter NSAIDs. Ther Clin Risk Manag. 2015;11:1061-1075.

28. Shaffer EE, Pham A, Woldman RL, Spiegelman A, Strassels SA, Wan GJ, Zimmerman T. Estimating the effect of intravenous acetaminophen for postoperative pain management on length of stay and inpatient hospital costs. Adv Ther. 2017;33(12):2211-2228.
29. Maiese BA, Pham AT, Shah MV, Eaddy MT, Lunacsek OE, Wan GJ. Hospitalization costs for patients undergoing orthopedic surgery treated with intravenous acetaminophen (IV-APAP) plus other IV analgesics or IV opioid monotherapy for postoperative pain. Adv Ther. 2017;34(2):421-435.

30. Elixhauser A, Steiner C, Harris DR, Coffey RM. Comorbidity measures for use with administrative data. Med Care. 1998;36(1):8-27.

31. ML M. Demystifying opioid conversion calculations. A guide for effective dosing. Bethesda, MD, 2010.

32. (APS) APS. Principles of analgesic use in the treatment of acute pain and cancer pain, 6th edition. Glenview, IL 60025,2008

33. van Buuren S, Boshuizen HC, Knook DL. Multiple imputation of missing blood pressure covariates in survival analysis. Stat Med. 1999;18(6):681-694.

34. Tan Z. Bounded, efficient and doubly robust estimation with inverse weighting. Biometrika. 2010;97:661-682.

35. Cameron AC, Trivedi PK. Microeconometrics: methods and applications. New York: Cambridge University Press, 2006.

36. Zou G. A modified poisson regression approach to prospective studies with binary data. Am J Epidemiol. 2004;159(7):702-706.

37. Oderda GM, Gan TJ, Johnson BH, Robinson SB. Effect of opioid-related adverse events on outcomes in selected surgical patients. J Pain Palliat Care Pharmacother. 2013;27(1):62-70.

38. Hansen RN, Pham A, Strassels SA, Balaban S, Wan GJ. Erratum to: comparative analysis of length of stay and inpatient costs for orthopedic surgery patients treated with IV acetaminophen and IV opioids vs. IV opioids alone for post-operative pain. Adv Ther. 2016;33(9):1646-1648.

39. Hansen RN, Pham A, Strassels SA, Balaban S, Wan GJ. Comparative Analysis of Length of Stay and Inpatient Costs for Orthopedic Surgery Patients Treated with IV Acetaminophen and IV Opioids vs. IV Opioids Alone for Post-Operative Pain. Adv Ther. 2016;33(9):1635-1645.

40. Apfel C, Jahr JR, Kelly CL, Ang RY, Oderda GM. Effect of i.v. acetaminophen on total hip or knee replacement surgery: a case-matched evaluation of a national patient database. Am J Health Syst Pharm. 2015;72(22):19611968.

41. Shah MV MB EM, Lunacsek O, Pham A, Wan GJ Hospitalization costs for patients undergoing orthopedic surgery treated with intravenous acetaminophen (IV-APAP) IV opioids or IV opioids alone for postoperative pain. Poster presented at the 2016 Annual Congress of Enhanced Recovery and Perioperative Medicine. Washington, DC, USA. 2016.

42. Shaffer E WR SA, Strassels S, Wan GJ, Zimmerman T. Estimating the effect of intravenous acetaminophen (IVAPAP) on length of stay and inpatient costs. Poster presented at the 41st Annual Regional Anesthesiology and Acute Pain Medicine Meeting. New Orleans, LA, USA. 2016.

43. Sinatra RS, Jahr JS, Reynolds LW, Viscusi ER, Groudine SB, Payen-Champenois C. Efficacy and safety of 
single and repeated administration of 1 gram intravenous acetaminophen injection (paracetamol) for pain management after major orthopedic surgery. Anesthesiology. 2005;102(4):822-831.

44. Singla NK, Parulan C, Samson R, Hutchinson J, Bushnell R, Beja EG, Ang R, et al. Plasma and cerebrospinal fluid pharmacokinetic parameters after single-dose administration of intravenous, oral, or rectal acetaminophen. Pain Pract. 2012;12(7):523-532.

45. Kerai S, Saxena KN, Taneja B. Post-caesarean analgesia: What is new? Indian J Anaesth. 2017;61(3):200-214.

46. Sutton CD, Carvalho B. Optimal pain management after cesarean delivery. Anesthesiol Clin. 2017;35(1):107-124.

47. Elia N, Lysakowski C, Tramer MR. Does multimodal analgesia with acetaminophen, nonsteroidal antiinflammatory drugs, or selective cyclooxygenase- 2 inhibitors and patient-controlled analgesia morphine offer advantages over morphine alone? Meta-analyses of randomized trials. Anesthesiology. 2005;103(6):1296-1304.

48. Maund E, McDaid C, Rice S, Wright K, Jenkins B, Woolacott N. Paracetamol and selective and non-selective non-steroidal anti-inflammatory drugs for the reduction in morphine-related side-effects after major surgery: a systematic review. Br J Anaesth. 2011;106(3):292-297. 